\title{
Jessie White Mario, una donna inglese tra i protagonisti del Risorgimento italiano
}

Roberta Barazza

\begin{abstract}
In questo articolo mi propongo di presentare una figura poco nota del Risorgimento italiano: Jessie White Mario, un'inglese, nata a Portsmouth nel 1832, interessata, fin dagli anni giovanili, alla lotta per l'indipendenza del nostro Paese. Conobbe personalmente e collaborò con Garibaldi e Mazzini nell'attività di propaganda e militanza politica. Fu infermiera di guerra in molte battaglie risorgimentali. Scrisse numerosi libri sulle figure del nostro Risorgimento e sui problemi sociali di quel tempo, e molti articoli per riviste italiane e straniere. Inglese, naturalizzata italiana, patriota e intellettuale impegnata, ingiustamente poco conosciuta, ebbe un ruolo importantissimo, accanto al marito, il patriota e studioso Alberto Mario di Lendinara, nella storia della nostra nazione.
\end{abstract}

Key words: Jessie White Mario. Alberto Mario. Garibaldi. Italia. Lendinara. Mazzini. Risorgimento.

Resumo: Nesse artigo me proponho a apresentar uma figura pouco conhecida do Ressurgimento italiano: Jessie White Mario, inglesa nascida em Portsmouth, no ano de 1832, e interessada desde cedo na luta pela independência do nosso país. Conheceu e colaborou com Garibaldie Mazzini nas atividades de propaganda política. Atuou nas batalhas como enfermeira e escreveu numerosos livros, além de artigos para revistas italianas e estrangeiras sobre os problemas sociais daquela época. Ingelsa, naturalizada italiana, injustamente pouco conhecida teve papel importante, a lado do marido, o patriota e intelectual Alberto Matio Lendinara, na história de nossa nação.

Palavras-chave: Jessie White Mario. Alberto Mario. Garibaldi. Italia. Lendinara. Mazzini. Ressurgimento. 
Il mio interesse per Jessie White Mario è sorto qualche anno fa quando insegnavo inglese in una scuola di Lendinara, in provincia di Rovigo. Non avevo mai sentito parlare di lei prima, e neanche del marito Alberto Mario che, nel Risorgimento italiano ha avuto un ruolo altrettanto importante. Pochi in Italia conoscono Jessie White Mario, solo qualche specialista o cultore di storia. Eppure questa donna inglese, naturalizzata italiana, ha dato un contributo importantissimo alla nascita del nostro Paese vivendo, lavorando, lottando per l'Unità d'Italia accanto ai personaggi più importanti dell`Ottocento italiano: Garibaldi, Mazzini, Cattaneo, Bertani e, soprattutto, Alberto Mario, con il quale 'intrecciò una lunga e intensa storia d'amore, forse la più bella del nostro Risorgimento"1. Carducci descrisse Jessie White come 'una grande donna a cui, lo scrivo senza enfasi, noi Italiani dobbiamo molto'. 2 Ma non è stato così; non siamo stati abbastanza grati né a lei e né a tutte le donne che hanno contribuito alla nascita e alla storia del nostro paese ${ }^{3}$. Pochi la conoscono; nei libri di storia non viene

${ }^{1}$ CIAMPI, Paolo. Miss Uragano. La donna che fece l'Italia. Firenze: Romano Editore, 2010, copertina. 2 . op. cit., copertina.

${ }^{3}$ Nel libro di Marina Cepeda Fuentes, Sorelle d'Italia. Le donne che hanno fatto il Risorgimento, Blu Edizioni, Torino, 2011, l'autrice ricostruisce la vita di alcune donne che hanno partecipato attivamente al Risorgimento italiano: tra queste Antonia Masanello, Eleonora de Fonseca Pimentel, Cristina Trivulzio di Belgioioso, Adelaide Bono Cairoli, Laura Solera Mantegazza, Giuditta Tavani Arcuati, la stessa Anita Garibaldi. Pochi italiani le conoscono e la memoria storica non è stata generosa con loro: rimane qualche strada a loro dedicata o qualche targa nei luoghi in cui hanno vissuto. Eppure il loro ruolo nel Risorgimento italiano è stato molto importante. Vorrei riportare qualche informazione sulla vita di alcune di loro perché sono figure ingiustamente poco conosciute e il tema della donna nella Storia è strettamente legato all'argomento centrale di quest'articolo dedicato a Jessie White. Antonia Masanello, giovane veneta, si travestì da uomo per poter partecipare alla spedizione dei Mille durante la quale dimostrò grande valore militare.Cristina Trivulzio di Belgioioso dal 1832, quando aveva solo 24 anni, animava a Parigi un importante salotto culturale e politico frequentato, tra gli altri, da Giuseppe Mazzini, Niccolò Tommaseo, Vincenzo Gioberti, Gioacchino Rossini, Vincenzo Bellini, Victor Hugo, George Sand, Stendhal, Frédéric Chopin, Franz Listz, Heinrich Heine. Eleonora Fonseca Pimental, vissuta a Napoli nel periodo pre-risorgimentale, fu tra i protagonisti della rivoluzione napoletana del 1799 contro i Borboni. Morì impiccata in seguito alla repressione della rivolta. La scrittrice e poetessa Innocenza Ansuini Tondi partecipò col marito, il patriota Ermenegildo Tondi, a tre tentativi insurrezionali contro lo Stato Pontificio, nel 1849, 1860 e 1867.La veneziana Teresa Perissinotti Manin organizzava l'equipaggiamento dei volontari che combattevano contro gli Austriaci. In Calabria ebbero un ruolo importante nella lotta contro i Borboni le combattive donne della comunità arbëresche, di origine albanese, stanziatesi nel sud d'Italia fin dal Quattrocento. Tra queste ricordiamo Maria Cucci detta 'Giubglia' che nascondeva, salvandoli, i patrioti ricercati dalla polizia. 
quasi mai citata; di lei resta qualche epigrafe e qualche strada che porta il suo nome. La Storia, ai tempi di Jessie White, era ancora Storia di uomini. E le cose non sono ancora sufficientemente cambiate.

Ciò che più mi affascina in Jessie White è la sua intelligenza, la sua volontà di capire, descrivere e intervenire negli eventi del suo tempo, il suo ruolo di intellettuale impegnata in un momento storico in cui pochissime donne studiavano, scrivevano, vivevano del proprio lavoro intellettuale. Già a Parigi, appena ventenne, iscritta alla facoltà di filosofia della Sorbonne, Jessie White si manteneva con i suoi racconti e i suoi articoli di giornale. E' un'attenta e curiosa testimone del suo tempo; dei successivi anni italiani ci lascerà un gran numero di articoli e libri e una ricca raccolta di documenti e testimonianze dell'inizio della storia unitaria del nostro Paese. Altre donne hanno partecipato al Risorgimento italiano, ma lei ha avuto soprattutto un ruolo di intellettuale impegnata, di giornalista e scrittrice sempre in prima linea, e in questo la sua esperienza è stata davvero straordinaria.

Affascina, in Jessie White, l'entusiasmo con cui decide di dedicarsi alla causa di un paese straniero. Elegge l'Italia a sua seconda patria. Molti patrioti italiani, come Mazzini, avevano trovato rifugio in Inghilterra e questo Paese, più che altri in Europa, sosteneva la causa della libertà dei popoli. Byron muore in Grecia lottando per l'indipendenza ellenica; Shelley dedica i suoi versi alla libertà dei popoli e degli individui. Mazzini vivrà esule in Inghilterra per molti anni, sempre circondato da amici che sostenevano la sua opera patriottica raccogliendo fondi per realizzarla. Anche Garibaldi viene calorosamente accolto in Inghilterra. Jessie White, già interessata alla storia italiana, coglie l'occasione di un viaggio a Nizza, con l'amica Emma Roberts, per conoscere personalmente Garibaldi. Il viaggio cambierà la sua vita. Rientrerà poi in Inghilterra con Emma, ma il suo futuro è deciso: tornerà in Italia e dedicherà la sua vita alla storia del nostro Paese.

Una aspetto interessante degli scritti di Jessie White è il suo sguardo 
di inglese sulle vicende italiane. Molti, nei suoi scritti, sono i confronti tra l'Inghilterra e l'Italia. E molti non sono affatto, per noi, lusinghieri. Jessie White è un'inglese che osserva il nostro Paese e spesso non riesce a trattenere lo sdegno per quanto vi avviene e per come le persone si comportano. I suoi giudizi sono a volte molto severi. Penso che questo confronto sia molto interessante. Vivere sempre nello stesso Paese comporta il rischio di assuefarsi e accettare ciò che, dall'esterno, può sorprendere o scandalizzare. I frequenti confronti di Jessie White tra la sua madre patria e la patria che si è scelta sono rivelatori delle differenze di vita e pensiero dell'Europa ottocentesca. E' partendo dalle sue personali esperienze in Inghilterra che Jessie non può trattenersi dal proporre cambiamenti e promuovere iniziative di carattere sociale e umanitario. E' curioso come certi suoi giudizi sulla società italiana, sui politici, sulle istituzioni, assomiglino molto a ciò che ancora oggi colpisce gli stranieri, specie nordeuropei: la corruzione, il malgoverno, il maschilismo, lo scarso ruolo sociale delle donne, l'apatia del popolo italiano, la drammatica povertà al sud, il progresso frenato da certi aspetti della religione, la retorica ampollosa dei discorsi ufficiali, lo scarso coraggio della stampa nel denunciare i potenti. Di certo non erano, e non sono, problemi solo italiani, ma anche oggi si pensa spesso, del Belpaese, ciò che Jessie White aveva acutamente colto e descritto.

\section{La vita di Jessie White Mario}

\subsection{Gli anni giovanili}

Jessie White nasce a Fort Inslet, vicino a Portsmouth, nel sud dell'Inghilterra. Il padre e il nonno paterno erano costruttori navali, proprietari di una prospera ditta molto nota nella zona. Il padre di Jessie, Thomas White, lavorava anche all'estero, in particolare in Italia e in Portogallo, dove si recava per progettare e costruire scali portuali e imbarcazioni. Thomas White, da bambino, era sempre stato bravo a scuola 
ma, a causa dell'avversione per un insegnante di latino, a 14 anni non concluse con successo l'anno scolastico; per questo motivo i genitori decisero che non avrebbe proseguito gli studi e lo mandarono invece a lavorare nella ditta paterna. Il padre di Jessie soffrì molto per ciò che lui considerava una grande ingiustizia, e si propose di non negare mai ai suoi figli la possibilità di studiare.

Thomas White si sposò tre volte e Jessie White nacque dal suo secondo matrimonio. Il primo si concluse nel 1826, con la morte della prima moglie Elizabeth, che aveva messo al mondo tre maschi e una femmina. Nel 1827 il padre di Jessie si risposa con Jane Teage Meriton, di origine americana, donna pratica e impegnata in famiglia e nel paese, dove organizzava scuole per i figli dei poveri.

Jessie White è figlia di Jane e nasce il 9 maggio $1832 \mathrm{ma}$, due soli anni dopo, sua madre muore. La terza moglie di Thomas White fu Jane Gain, una delle maestre delle scuole di Jane White. Jessie White ebbe quindi per madre effettiva Jane Gain.

In casa White si respirava un'aria di grande severità morale e religiosa. Il padre di Jessie era un puritano rigoroso ed esercitò sulla sua famiglia un'influenza molto forte. Fred, fratello di Jessie, divenne, da giovane, un fanatico religioso; Jessie invece sviluppò, fin da bambina, una forte insofferenza per quell'eccessivo rigore e divenne una ribelle agnostica, per niente interessata alle pratiche religiose. In famiglia era considerata disobbediente e poco femminile. Tuttavia, anni più tardi, Jessie descrisse la sua infanzia come un periodo sereno in cui poté esprimersi con una certa libertà. Intorno ai dieci anni fu profondamente affascinata dal predicatore del suo paese, John Daniel Morell, di cui la entusiasmavano i sermoni tolleranti ed eclettici. Morell fu allontanato dalla parrocchia proprio per la modernità e libertà del suo pensiero, formatosi più su studi filosofici che teologici. 
Durante gli anni di studio a Reading, Jessie si rivelò una studente entusiasta e vivace, interessata soprattutto alla letteratura inglese: anni dopo, anche nei suoi scritti sociali e politici sull'Italia, ricorrono citazioni da Shelley e Byron, i suoi poeti preferiti.

Jessie proseguirà gli studi a Londra e a Birmingham. Qui conosce e frequenta un altro predicatore mal tollerato nei circoli più conservatori della città, ma apprezzato e sostenuto dalle menti più moderne e sensibili ai problemi sociali: George Dawson, non a caso amico di Morell, il cui liberalismo e impegno sociale al contempo scandalizzavano i devoti delle parrocchie e entusiasmavano le menti più vivaci e aperte, come quella di Jessie White. Dawson mal rappresentava l'immagine tradizionale del teologo attento soprattutto alle questioni dottrinali. Egli credeva che il compito di un ministro della Chiesa fosse quello di trasformare la conoscenza delle Sacre Scritture in un lavoro concreto che migliorasse i rapporti tra la gente, la vita quotidiana delle persone e le istituzioni. Fece costruire scuole e biblioteche, condannò la schiavitù, promosse l'educazione e i diritti delle donne, difese i diritti dei lavoratori e propose la rieducazione e il reinserimento dei criminali.

Questi erano temi importanti per Jessie: fin dai suoi primi scritti trapela la volontà di impegnarsi per migliorare la vita delle persone e l'organizzazione delle società. I suoi primi racconti, di quand'era studentessa a Birmingham e poi alla Sorbonne, non rivelano grande talento letterario quanto piuttosto un profondo interesse, idealista e pragmatico allo stesso tempo, per l'impegno sociale e politico.

\subsection{Jessie sceglie l'Italia come sua seconda patria}

L'interesse di Jessie White per l'Italia crebbe con la frequentazione dei circoli italiani di Londra. Nel 1855 non si lasciò sfuggire l'occasione di accompagnare l'amica Emma Roberts a Nizza per incontrare niente meno che Giuseppe Garibaldi. Rimase affascinata dall'Eroe dei due Mondi e 
quando ritornò in Inghilterra, nel 1855, aveva già idee chiare sul suo futuro: si sarebbe dedicata alla nascita della nazione italiana, indipendente e unificata.

A Londra frequentò i circoli che sostenevano i patrioti italiani, come quello della famiglia Ashurst. Anche Giuseppe Mazzini frequentava gli Ashurst e fu proprio nella loro casa che Jessie White lo conobbe e ne rimase conquistata: era per lei un grande personaggio carismatico che avrebbe cambiato le sorti del Paese, una sorta di santo, un idealista, dall'altissimo profilo morale, che credeva che un cambiamento storico fosse possibile solo attraverso l'educazione del popolo e il suo coinvolgimento nella lotta politica.

L'amicizia e la frequentazione di Mazzini e Garibaldi sono stati determinanti per il coinvolgimento della giovane inglese nella lotta politica italiana. Fin dall'inizio, il punto di riferimento politico del suo pensiero politico fu Mazzini, di cui ammirava l'idealismo, la devozione totale al programma politico e i principi repubblicani. Secondo Mazzini non spettava alla monarchia sabauda il compito di guidare il nascente stato unitario. Solo un regime repubblicano avrebbe potuto rappresentare la volontà del popolo italiano.

Quando, nel 1855, Jessie White progettava di partecipare alle campagne risorgimentali, decise che avrebbe potuto farlo lavorando come infermiera. Per questo nel 1856 chiese l'ammissione alla facoltà di medicina dell'università di Londra, ma le fu negato. Perché era una donna. Denunciò il fatto ma non servì a molto. "Non andrò comunque in America", scrisse infine Jessie White, “ per seguire quegli studi che l'Inghilterra mi rifiuta. Ma per varie ragioni sono contenta di aver fatto quanta esperienza. Diverse personalità nel campo medico mi hanno assicurato che se un gruppo di donne chiedesse oggi l'ammissione in uno o più ospedali, dopo il vespaio suscitato tra gli spiriti più aperti dalla mia richiesta, sarebbe molto 
improbabile che la loro domanda venisse respinta ..."4

Questo è davvero un aspetto fondamentale della sua personalità: ciò che le importa è che le sue azioni servano a migliorare la società. Può lavorare come infermiera o giornalista, aiutare i figli dei poveri, insegnare o scrivere libri: aldilà dei diversi ruoli di volta in volta assunti, il leitmotif che lega le sue esperienze è la volontà di aiutare le persone, migliorare le loro condizioni di vita, cambiare la società in senso civile, culturale $\mathrm{e}$ democratico, denunciare ingiustizie e sofferenze. I suoi articoli di giornale e i suoi saggi, il suo lavoro di militante, infermiera o insegnante hanno questo fine comune: contribuire al progresso della società.

\subsection{Alberto Mario}

Nel 1857 Jessie White si spostava di città in città per diffondere il pensiero mazziniano, far proseliti e raccogliere fondi per il programma politico. Si trovava a Genova con Mazzini quando questi le presentò Alberto Mario, di cui era ospite.

Alberto Mario era nato nel 1825 a Lendinara, in Polesine. A diciannove anni si era iscritto all'Università di Padova, prima alla facoltà di matematica e poi a quella di legge, ma non aveva potuto concludere gli studi perché la sua partecipazione alle cospirazioni politiche lo avevano inviso alla autorità austriache che allora governavano il Regno Lombardo-Veneto. Dovette lasciare il Veneto e visse prima a Bologna, dove sperava di riprendere gli studi, e poi a Milano, Firenze e Genova. In quest'ultima città aveva messo la sua casa a disposizione di Mazzini, che in Italia viveva in incognito. Alberto Mario condivideva solo in parte il programma politico mazziniano e, col passare del tempo, le loro posizioni si distanziarono ulteriormente. Fu Mazzini colui che presentò Jessie ad Alberto chiedendogli di accompagnarla nei suoi impegni di militante.

Erano i giorni in cui Pisacane stava preparando un complotto che

${ }^{4}$ BODICHON, Barbara Leigh Smith. Women and Work, London: Bosworth \& Harrison, 1857, p. 53-56. 
mirava a rovesciare il governo borbonico di Napoli. Pisacane aveva progettato di imbarcarsi su una nave, a Genova, prenderne il controllo e dirottarla a Napoli, dove sperava che il popolo si unisse in un'insurrezione armata contro la monarchia borbonica. Il piano fallì, Pisacane fu ucciso e molti patrioti furono accusati di aver sostenuto il complotto. Tra questi Jessie White e Alberto Mario. Il 4 luglio 1857 entrambi furono rinchiusi nel carcere di Genova. Alberto vi rimase due mesi, Jessie quattro. Interrogata, negò di aver partecipato alla cospirazione; in realtà l'aveva propagandata e indirettamente sostenuta.

Proprio in carcere, come in un romanzo avventuroso, Jessie e Mario si conosceranno e apprezzeranno tanto da decidere di sposarsi, non appena fossero tornati liberi. I due prigionieri si scambiarono lettere e messaggi personali. Comprendendo l'intelligenza e l'impegno di Jessie, Alberto decide di farle conoscere molti aspetti della storia e della cultura italiana. Scarcerati entrambi, quattro mesi dopo, si resero però conto che sposarsi non sarebbe stato facile per loro. Dove avrebbero abitato? Per Alberto era vietato il ritorno a Lendinara e, in quanto cospiratore, non gli sarebbe stato facile trovare un lavoro stabile neanche nel resto del Paese. Cosa poteva offrire a sua moglie? D'altro lato non desiderava trasferirsi in Inghilterra dove non avrebbe potuto continuare i progetti politici che, peraltro, interessavano entrambi. Infine accettarono di partire per Portsmouth. Non avevano scelta perché a Jessie era stato concessa la scarcerazione solo a patto che lasciasse entro cinque giorni il Paese. Il padre di Jessie, inoltre, preoccupato per le sorti della figlia, insisteva perché facesse ritorno a Portsmouth. Decisero così di partire. Alberto fu subito accettato e apprezzato dalla famiglia White. Si sposarono in Inghilterra il 19 dicembre 1857, con rito civile. Grazie anche all'aiuto di Mazzini, trovarono casa nella capitale e ripresero la loro attività culturale e politica: Alberto scriveva articoli e libri sulla cultura e la politica italiana e Jessie li traduceva in inglese. Presto Jessie riprende anche a tenere 
conferenze, sulla causa italiana, in Inghilterra e in Scozia. Alberto è sorpreso dal grande affetto e sostegno che molti, in Gran Bretagna, offrivano al popolo italiano.

Nonostante le molte difficoltà, il matrimonio dei Mario fu un'unione felice. Non si può certo dire che rappresentasse il tipico ménage famigliare di una coppia ottocentesca, anzi la loro era un'unione molto speciale, basata su affinità intellettuali, culturali, politiche, e un impegno comune di lavoro per la realizzazione di grandi progetti storici. Il loro affetto cresceva insieme al rispetto e all'interesse per il lavoro l'uno dell'altro, e per le scelte politiche e intellettuali che a volte divergevano. Ecco cosa scrive Elizabeth Adams Daniels nel suo saggio dedicato a Jessie White: "Nonostante la cornice romantica, era un matrimonio tra uguali. Un matrimonio nel senso moderno della parola molto prima che la Nora di Ibsen sbattesse la porta di passaggio al mondo esterno. Doveva continuare a essere un matrimonio ambulante, anticonvenzionale e molto interessante, durato più di un quarto di secolo, fino alla morte di Alberto." 5

Nel 1858 i coniugi Mario decisero di realizzare un viaggio in America, da tempo progettato. Negli USA molti circoli culturali si interessavano alla causa dell'indipendenza italiana. A New York Jessie tenne conferenze e raccolse fondi per i patrioti. Scrisse articoli per giornali americani e, soprattutto, ottenne un incarico fisso come corrispondente dall'Italia per 'Nation', il che garantì alla coppia una certa stabilità economica negli anni seguenti.

Quando, dopo otto mesi in America, i Mario ritornarono in Italia, si stavano preparando gli eventi decisivi per la nascita della nostra nazione. In Veneto, dove cercarono di raggiungere la casa, a Lendinara, del padre di Alberto, gravemente malato, furono arrestati e imprigionati. La stesso avvenne, poco tempo dopo, a Bologna. Dovettero allontanarsi, prima verso

${ }^{5}$ DANIELS, Elizabeth Adams. Posseduta dall'angelo. Jessie White Mario, la rivoluzionaria del Risorgimento. Milano: Mursia, 1977. p. 84. 
Lodi e poi a Lugano, in Svizzera, dove rimasero per vari mesi, fino a maggio 1860.

Fervevano intanto i preparativi per lo sbarco in Sicilia e la liberazione del sud della penisola. L'11 maggio 1860, partendo da Quarto, nei pressi di Genova, la nave dei Mille di Garibaldi raggiunse la Sicilia. I Mario, informati dell'evento, lasciarono Lugano e, l'11 giugno, sbarcarono anch'essi nell'isola. In quell'occasione Alberto conobbe personalmente Garibaldi. Ecco come egli stesso descrive l'evento: "Nel 1860 sbarcammo con Medici a Castellammare ed arrivammo ad Alcamo. Garibaldi ci venne incontro a cavallo... Non gli ero ancora mai stato presentato personalmente, ma subito mi prese la mano dicendo: 'Voi siete Alberto Mario; sono contento di avervi qui, siate il benvenuto'. Aveva indovinato chi ero perché ero accompagnato da mia moglie. Mise la sua carrozza a nostra disposizione e ritornammo con lui a Palermo."

A luglio 1860 i garibaldini avevano già conquistato l'intera Sicilia. Alberto Mario partecipò alla spedizione guidando un reggimento di soldati, organizzando le nuove amministrazioni locali, fondando scuole militari. Jessie, invece, vi partecipò come infermiera di guerra, ma tutto ciò che osservava e viveva personalmente divenne poi materiale per i suoi articoli e i suoi libri. Quando le 'Camicie Rosse' raggiunsero Napoli, Garibaldi affidò ad Alberto Mario l'incarico di far proseliti per la causa garibaldina e arruolare nuovi soldati. Anche Napoli fu presto conquistata. Il 17 marzo 1861 fu proclamata la nascita del Regno d'Italia. Roma non vi faceva ancora parte, né il Triveneto. Il Risorgimento non si era ancora concluso, ma una nuova nazione era nata. Jessie e Alberto Mario erano stati tra i suoi principali protagonisti.

\subsection{Dopo l'Unificazione}

Negli anni successivi all'unificazione italiana i Mario non ebbero vita ${ }^{6}$ MARIO, Alberto. Personal Reminiscences of General Garibaldi. Macmillan's Magazine. v. 46
(luglio 1882), pp. 245-256. 
facile. Se fino ad allora il loro compito era chiaro, lavorare per la nascita della nazione italiana, dopo il 1861 si trovarono per vari mesi senza obiettivi precisi. Soggiornarono a Milano, in Svizzera, in Inghilterra, per poi tornare in Italia. Impossibile per loro vivere a Lendinara perché il Veneto non era ancora stato liberato dagli austriaci. Jessie White continuava a scrivere per riviste americane e inglesi, mentre Alberto si interessava soprattutto dell'evolversi della situazione politica. In seguito alla morte del padre, Jessie allenterà ulteriormente i contatti con la sua madre patria. Garibaldi si recherà ancora una volta in Inghilterra, accolto trionfalmente. Negli anni seguenti parteciperà alle battaglie per la conquista del Veneto, che entrerà a far parte del Regno d'Italia nel 1866. In seguito, mal sopportando l'esitazione con cui si affrontava la questione romana, attaccò Roma senza attendere l'approvazione del governo italiano e del re Vittorio Emanuele II. Si scontrò per questo con l'esercito italiano, cadde ferito e venne arrestato. Il 20 settembre 1870, in seguito all'intervento dell'esercito italiano guidato dal Generale Cadorna, anche lo Stato Pontificio viene annesso al Regno d'Italia.

Garibaldi parteciperà a un'ultima impresa militare, in Francia, in difesa della Terza Repubblica minacciata dall'invasione dell'esercito prussiano. Alberto Mario rifiuta di sostenere Garibaldi in quest'impresa non dimenticando quanto la Francia abbia ostacolato il processo di unificazione italiana. Jessie invece accetta di parteciparvi nel ruolo di corrispondente di guerra. Da questa esperienza deriveranno vari articoli per 'Tribune' di New York e 'Scotsman' di Edimburgo, e il saggio I garibaldini in Francia, pubblicato nel 1871.

Negli anni successivi all'unificazione italiana i Mario ebbero pesanti difficoltà economiche. Entrambi scrivevano libri e articoli di giornali per varie testate italiane e straniere, ma i proventi non erano cospicui. Vissero a Roma e a Firenze finché, nel 1871, Alberto Mario ereditò da uno zio una casa a Lendinara, e qui la coppia si stabilì per alcuni anni. Nonostante le ristrettezze economiche, non rinunciarono a viaggiare per poter continuare le loro ricerche e i loro scritti. Jessie si recò più volte al sud per studiare, tra l'altro, le durissime condizioni di vita dei napoletani e dei siciliani di cui parla 
in La Miseria di Napoli e Le miniere di zolfo.

Già nei tardi anni '60 Alberto Mario si ammala di cancro alla faringe. La coppia risiede da tempo a Lendinara quando, nel 1883, dopo anni di cure, egli viene a mancare. Sarà sepolto a Lendinara dove ancora oggi si trova la sua tomba, accanto a quella della moglie. Molti dei suoi scritti, lasciati incompiuti, saranno portati a termini da Jessie, che gli sopravviverà di una ventina d'anni.

Negli ultimi anni Jessie White si dedicherà, tra l'altro, alle biografie dei grandi uomini del Risorgimento: Garibaldi, pubblicato nel 1879, Della vita di Giuseppe Mazzini del 1886, Agostino Bertani e i suoi tempi nel 1887. Pubblica inoltre In memoria di G. Nicotera, Camicia Rossa, La mente di Carlo Cattaneo e Scritti letterari e artistici in cui raccoglie e integra gli scritti del marito. Sono solo alcuni dei suoi libri più noti. A causa delle difficoltà economiche, dal 1896, quando aveva 64 anni, fino alla morte, accetta l'incarico di insegnare letteratura inglese presso la Facoltà di Pedagogia della Scuola Normale di Firenze. La sua morte avviene, in quella stessa città, il 5 marzo 1906. Le sue ceneri sono conservate a Lendinara, accanto alla tomba del marito.

\section{Bibliografia}

BIAGIANTI , Ivo (a cura di). La Nuova Italia' nelle corrispondenze americane di Jessie White Mario (1866-1906). Firenze: Centro Editoriale Toscano, 1999.

FUENTES, Marina Cepeda. Sorelle d'Italia. Le donne che hanno fatto il Risorgimento. Torino: Blu Edizioni, 2011.

CIAMPI, Paolo. Miss Uragano, la donna che fece l'Italia. Firenze: Romano Editore, 2010.

DANIELS, Elizabeth Adams. Posseduta dall'angelo. Jessie White Mario, la rivoluzionaria del Risorgimento. Milano: Mursia, 1977.

PRISCO, Mario. Adorabile Uragano. Dalle lotte risorgimentali alla 'Miseria di Napoli. La straordinaria avventura di Jessie White Mario. Napoli: Stamperia del Valentino, 2011.

MARIO, Jessie White. La miseria di Napoli. Napoli: Imagaenaria, 2005. 